Research Paper

\title{
Long Noncoding RNA LINC00467 Promotes Glioma Progression through Inhibiting P53 Expression via Binding to DNMT1
}

Yin Zhang1,2\#, Xuefeng Jiang2\#, Zhisheng $\mathrm{Wu}^{2}$, Daling Hu², Junli Jia², Jinfeng Guo², Tian Tang2, Jialin $\mathrm{Yao}^{2}$, Hongyi Liu ${ }^{\natural}$, Huamin Tang ${ }^{2 \bowtie}$

1. Department of Neurosurgery, Sir Run Run Hospital, Nanjing Medical University.

2. School of Basic Medical Sciences, Nanjing Medical University.

3. Department of Geriatrics, Sir Run Run Hospital, Nanjing Medical University.

4. Department of Neurosurgery, The Affiliated Brain Hospital of Nanjing Medical University.

\#These authors contributed equally to this work.

$\triangle$ Corresponding authors: Huamin Tang, E-mail: htang@njmu.edu.cn. School of Basic Medical Sciences, Nanjing Medical University, Nanjing 210008, China; Hongyi Liu, E-mail: hyliu18@126.com. Department of Neurosurgery, The Affiliated Brain Hospital of Nanjing Medical University, Nanjing 210029, China.

(C) The author(s). This is an open access article distributed under the terms of the Creative Commons Attribution License (https://creativecommons.org/licenses/by/4.0/). See http://ivyspring.com/terms for full terms and conditions.

Received: 2019.11.09; Accepted: 2020.02.06; Published: 2020.03.04

\begin{abstract}
Purpose: This study aimed to investigate whether long noncoding RNA (IncRNA) LINC00467 could regulate proliferative and invasive abilities of glioma cells via p53 and DNA methyltransferase 1 (DNMT1), so as to participate in the occurrence and progression of glioma. Methods: LINC00467 expression in glioma was analyzed by GEPIA database and LINC00467 expression in glioma cell lines was detected by qRT-PCR. The regulatory effects of LINC00467 and p53 on proliferative, invasive capacities and cell cycle were conducted by CCK-8 and EdU assays, transwell assay and flow cytometry, respectively. The binding conditions between LINC00467, DNMTI and p53 were determined by RNA immunoprecipitation (RIP) and Chromatin immunoprecipitation (ChIP) assays. Western blot was conducted to determine whether LINC00467 could regulate $\mathrm{p} 53$ in glioma cells. Finally, rescue experiments were carried out to evaluate whether LINC00467 regulates proliferative and invasive abilities of glioma cells through p53. Results: The expression of LINC00467 was significantly up-regulated in tumor samples than that in normal samples, which was not correlated with patient survival time. Besides, expression of LINC00467 was higher in glioma cells than that of negative control cells. Upregulation of LINC00467 promoted proliferative and invasive abilities, and accelerated cell cycle in G0/G1 phase of U87 and LN229 cells. The results of RIP and ChIP assays demonstrated that LINC00467 could bind to DNMT1 and inhibit $\mathrm{p} 53$ expression. Overexpression of $\mathrm{p} 53$ partially reversed the enhancement of LINC00467 on proliferative and invasive abilities of glioma cells. Conclusion: These results indicated that high expression of LINC00467 could promote proliferative and invasive abilities of glioma cells through targeting inhibition of p53 expression by binding to DNMT1.
\end{abstract}

Key words: LINC00467; Glioma; Proliferation; Invasion; DNMT1; P53

\section{Introduction}

Glioma represents the most frequent intracranial malignant brain tumors in adults, characterized by rapid growth, early metastasis and high lethality [1,2]. Despite great progress on glioma treatment that has been made, the prognosis of glioma still remains grim [3]. Mounting researches have been performed to detect the potential mechanisms associated with glioma, only a few have been revealed so far $[4,5]$. 
Therefore, further exploring the potential mechanisms of these genomic changes in glioma is urgently required.

Long noncoding RNAs (lncRNAs) are a type of transcript that is longer than 200 nucleotides in length with restricted protein-coding capacity [6-8]. It has been reported that the expression levels of lncRNAs were dysregulated in various tumors $[9,10]$. Mounting evidence exhibits that lncRNAs are the key regulators in several cellular and biological processes including cellular proliferation, metastasis, and cell apoptosis. For examples, lncRNA small nucleolar RNA host gene 20 (lncSNHG20) is upregulated and can promote cell proliferation and migration in non-small cell lung cancer [11], lncRNA Growth Arrest Specific 5 (lnc GAS5) differentially regulates cell cycle arrest and apoptosis in human neuroblastoma through activating breast cancer type 1 susceptibility protein (BRC A1) and p53 [12], H19 can promote tumor growth and indicate a poor prognosis in colorectal cancer [13]. Although lncRNAs are regarded as candidate therapeutic targets, the role they play on glioma is little known.

LINC00467 is a novel lncRNA localized at the chromosomal locus 1q32.3, and has been reported to be markedly dysregulated in hepatocellular carcinoma [14], lung adenocarcinoma [15] and neuroblastoma [16]. Additionally, it has been confirmed that LIN C00467 could promote cell proliferation, migration and invasion of lung adenocarcinoma cells [17]. However, the specific mechanism of LINC00467 in glioma remains to be further investigated.

In this study, we discovered that LINC00467 expression was increased in glioma tissues and cell lines, and overexpression of LINC00467 could significantly promote cell proliferation and invasion in vitro. Additionally, flow cytometric analysis indicated that upregulation of LINC00467 promoted cell cycle and inhibited cell apoptosis. Furthermore, we found that LINC00467 could bind to DNMT1 and inhibit the expression of p53. Taken together, all findings suggested that LINC00467/DNMT1/p53 regulatory axis might be involved in the occurrence of glioma.

\section{Materials and methods}

\section{GEPIA database}

GEPIA database (http://GEPIA.cancer-pku.cn/ index.html) was used to assess the expression level of LINC00467 and survival prognosis of glioma patients. The database can be used to analyze differential genes in tumors, including gene co-expression analysis, gene OS and DFS survival curve analysis, and gene and clinical stage correlation analysis.

\section{Cell lines and culture conditions}

Human glioma cell lines LN229, LN308, U87, LN229 and human normal glial cell line HEB were purchased from American Type Culture Collection (ATCC, Manassas VA, USA). All cells were cultured in RPMI-1640 medium supplemented with $10 \%$ fetal bovine serum (Life Technologies, USA), $100 \mathrm{U} / \mathrm{ml}$ penicillin and $100 \mu \mathrm{g} / \mathrm{ml}$ streptomycin (Gibco, NY, USA). Cells were incubated at $37^{\circ} \mathrm{C}$ in humidified air containing $5 \% \mathrm{CO}_{2}$. Cell passage was performed using trypsin until $80 \%-90 \%$ of confluence.

\section{Cell transfection}

Small interference RNAs (siRNAs) or overexpressed plasmid for LINC00467, DNMT1 and p53 and corresponding negative controls were synthesized by GenePharma (Shanghai, China) and transfected into the cells to a final oligonucleotide concentration of $10 \mathrm{nmol} / \mathrm{l}$. Transfection was performed using Lipofectamine 2000 (Invitrogen, Carlsbad, CA, USA) in accordance with the manufacturer's instructions. The cells were tested after $48 \mathrm{~h}$ of transfection.

\section{RNA extraction and quantitative real-time polymerase chain reaction}

Total RNA was extracted from cells through using TRIzol reagent (Invitrogen, Carlsbad, CA, USA) in accordance with the manufacture's guide. Then Reverse Transcription Kit (Takara, Tokyo, Japan) was utilized to reversely transcribe RNA into cDNA. Quantitative real-time polymerase chain reaction (qRT-PCR) was carried out to detect the mRNA expression by utilizing SYBR® Premix Ex TaqTM (Takara) on the ABI 7500HT (Applied Biosystems, Foster City, CA, USA) following the protocols. The expression of mRNA was normalized to the Glyceraldehyde-3-phosphate dehydrogenase (GAPDH) expression level. Each experiment was replicated thrice, and the relative expression was calculated utilizing the $2^{-\Delta \Delta C t}$ method. All primers involved are listed in Table 1.

\section{Cell viability assay}

Cell-Counting Kit 8 (CCK8; Dojindo Laboratories, Kumamoto, Japan) reagent was utilized to analyze the proliferative capabilities of U87 and LN229 cells which received different treatments. After treatments, exponentially growing cells $\left(3 \times 10^{3}\right.$ per well $)$ were plated into 96-well culture plates. After cells were cultured for 1 day, 2 days, 3 days, 4 days, 5 days, 6 days, respectively, CCK8 solution $(10 \mu \mathrm{L})$ was added to each well following the manufacturer's instructions. After the cells were incubated at $37^{\circ} \mathrm{C}$ for an additional $1 \mathrm{~h}$, the OD value at $450 \mathrm{~nm}$ was recor- 
ded utilizing a microplate reader. Each assay was repeated in triplicate.

Table 1. Sequences of primers for qRT-PCR

\begin{tabular}{|c|c|c|}
\hline Name & & Sequence \\
\hline \multirow[t]{2}{*}{ LINC00467 } & Forward & 5'- GCCAGAGCAAGACTCTGTCTAC - $3^{\prime}$ \\
\hline & Reverse & 5'-GATGGGATACACATTCAATCAT -3' \\
\hline \multirow[t]{2}{*}{ p15 } & Forward & 5'-GAAGAATCCAACAACGGC-3' \\
\hline & Reverse & 5'-TCACAATCAGGGAAGCAT-3' \\
\hline \multirow[t]{2}{*}{ GAPDH } & Forward & $5^{\prime}$-GCACCGTCAAGGCTGAGAAC-3' \\
\hline & Reverse & 5'-GGATCTCGCTCCTGGAAGATG-3' \\
\hline \multirow[t]{2}{*}{ p21 } & Forward & 5'- CGACGCGTCGTTGTAATAAAGCCTCCAG -3' \\
\hline & Reverse & 5'- GACTAGTCGTTTTCAT TTCAATCGTAG -3' \\
\hline \multirow[t]{2}{*}{ p27 } & Forward & 5'- TACTGGCACCACTGGAAACC - $3^{\prime}$ \\
\hline & Reverse & 5'-GACCACTGAGGTTAGAGCCA-3' \\
\hline \multirow[t]{2}{*}{ p53 } & Forward & 5'- CTTGTGCCCTGTGAGGTCGTTGA - $3^{\prime}$ \\
\hline & Reverse & 5'-GACCACTGAGGTTAGAGCCA-3' \\
\hline \multirow[t]{2}{*}{ DNMT1 } & Forward & $5^{\prime}$ - TGGGAACTATATCTCTCGCTTGC - $3^{\prime}$ \\
\hline & Reverse & 5'- GGGTGAGACAGAACCGTCT - $3^{\prime}$ \\
\hline \multirow[t]{2}{*}{ U6 } & Forward & 5'- GCTTCGGCAGCACATATACTAAAAT-3' \\
\hline & Reverse & 5'- CGCTTCACGAATTTGCGTGTCAT - $3^{\prime}$ \\
\hline
\end{tabular}

\section{Ethynyl deoxyuridine (EdU) assay}

Proliferating cells were determined by using the 5-ethynyl-2'-deoxyuridine (EdU) abeling/detection kit (Ribobio, Guangzhou, China) according to the manufacturer's protocol. The transfected LN229 and U87 cells were treated with $50 \mu \mathrm{mol} / \mathrm{L}$ of EdU for 2 hours at $37^{\circ} \mathrm{C}$ and then the cultured cells were fixed with $4 \%$ paraformaldehyde for 30 minutes and stained with $1 \times$ Apollo reaction cocktail for 30 minutes before being incubated with $100 \mu \mathrm{L}$ of Hoechst33342 at $5 \mu \mathrm{g} / \mathrm{mL}$ for 30 minutes. The percentage of EdU positive cells was examined using a fluorescent microscope. All the assays were repeated for three times.

\section{Cell invasion assay}

For cell invasion assay, transfected cells $\left(1 \times 10^{5}\right)$ were cultured in the upper invasion chambers $(8 \mathrm{um}$ pore size, Millipore Corporation, Billerica, MA) coated with Matrigel RPMI-1640 medium without serum, while $600 \mu \mathrm{l}$ of medium supplemented with $10 \%$ FBS was added to the lower chamber. After 48 hours, the cells which couldn't migrate through the chamber were removed. The cells from the lower chamber were fixed using $4 \%$ paraformaldehyde for 30 minutes, and stained using crystal violet for 20 minutes. Penetrating cells were captured in 5 randomly selected fields of each sample. All the assays were conducted three times independently.

\section{Cell cycle assay}

At 48 hours after transfection, U87 and LN229 cells were harvested and washed using ice-cold PBS solution. Subsequently the cells were fixed with $70 \%$ ethanol overnight at $4{ }^{\circ} \mathrm{C}$ before being re-suspended using propidium iodide (PI)/RNase A solution (5 $\mu \mathrm{g} / \mathrm{mL}$ PI and $100 \mathrm{mg} / \mathrm{mL}$ RNase A) and incubated for $15 \mathrm{~min}$ at room temperature in the dark. Then the flow cytometer (Millipore Guava) was utilized to analyze cell cycle. All the assays were conducted three times independently.

\section{Cell apoptosis assay}

The cells were harvested $48 \mathrm{~h}$ after the transfection and washed twice using cold PBS. Then cells were re-suspended in binding buffer (BD Biosciences). Annexin V-FITC was utilized to stain the cells which were then resuspended using binding buffer $(100 \mu \mathrm{L})$, before $5 \mathrm{~mL}$ of allophycocyanin-annexin $\mathrm{V}$ (BD Biosciences) and $50 \mathrm{mg} / \mathrm{mL}$ propidium iodide (Invitrogen) were added to it. Subsequently the cells were mixed and incubated for $15 \mathrm{~min}$ in the dark at room temperature. Flow cytometry (Millipore Guava) was used to detect and quantify the apoptotic cells based on the manufacturer's instructions.

\section{Dual-luciferase reporter assay}

Briefly, glioma cells were plated in 24-well plates, cultured overnight, and transfected using Lipofectamine 2000 according to the manufacturer's protocol. After transfection for $24 \mathrm{~h}$, Firefly and Renilla luciferase activities were measured using the dual-luciferase reporter assay system (Promega, Madison, WI, USA). The ratios of luminescence from Firefly to Renilla luciferase were normalized through three independent experiments.

\section{Subcellular fractionation location}

Nuclear and cytoplasmic RNA were extracted utilizing the PARIS Kit (Life Technologies, USA) following its manufacturer's guides. Then we performed qRT-RCR to detect relative RNA level isolated from each fraction. U1 acted as nuclear control transcript, while GAPDH functioned as cytoplasmic marker.

\section{RNA-binding protein immunoprecipitation assay}

RNA-binding protein immunoprecipitation (RIP) assay was conducted on U87 and LN229 cells by using EZ-Magna RIP Kit (Millipore, Billerica, MA, USA) following the manufacturer's instructions. The transfected cells were washed with ice-cold PBS and then mixed with an equivalent volume of RIP lysis buffer. Next, the lysis products were incubated with 5 $\mu \mathrm{g}$ of human anti-DNMT1 antibody (Millipore, Billerica, MA, USA) or negative control mouse IgG (Millipore, Billerica, MA, USA) for $2 \mathrm{~h}$ at $4{ }^{\circ} \mathrm{C}$. Subsequently, each sample was mixed with $50 \mu \mathrm{L}$ of prepared magnetic beads and incubated at $4{ }^{\circ} \mathrm{C}$ overnight. The beads were briefly washed (five times in total) with RIP buffer and resuspended in $500 \mu \mathrm{L}$ of 
A

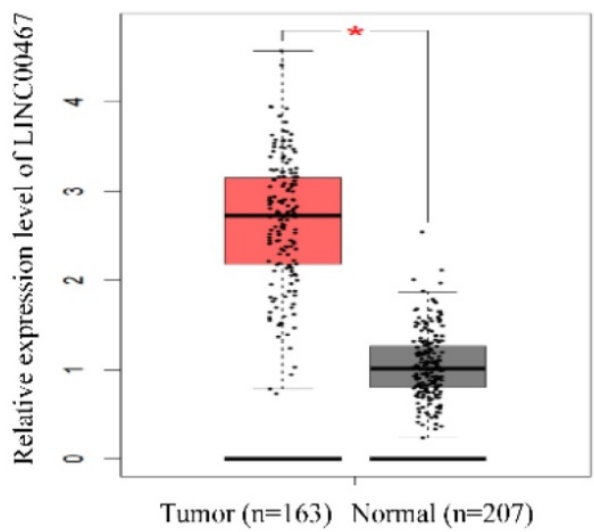

$\mathrm{B}$

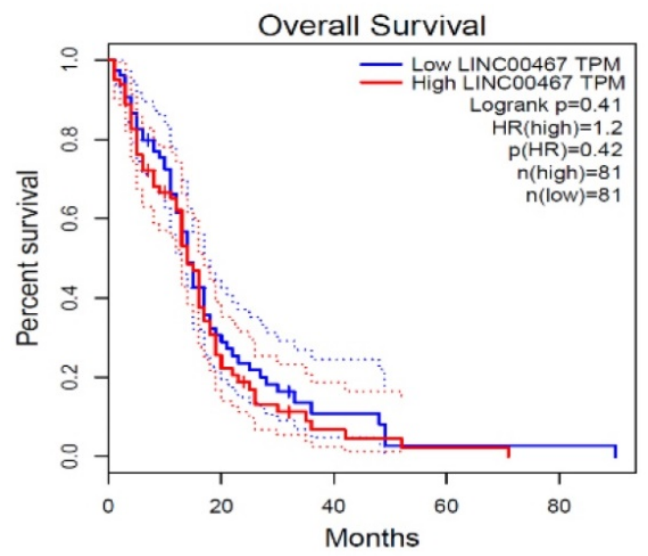

Figure 1. LINC00467 expression is remarkedly increased in glioma A. LINC00467 levels in glioma tissues ( $\mathrm{n}=163)$ and normal tissues ( $\mathrm{n}=207)$ analyzed in TCGA STAD database. B. The Kaplan-Meier curve depicts the overall survival of 162 patients with glioma. Error bars indicate mean \pm standard errors of the mean. *P < 0.05 . TCGA, the Cancer Genome Atlas.

TRIzol LS (Life Technology, Carlsbad, CA, USA). Finally, purified RNA was subjected to qRT-PCR analysis.

\section{Chromatin immunoprecipitation (ChIP) assay}

EZ-ChIP kit (Millipore) was utilized to perform ChIP assays following its manufacturer's protocol. The cells were fixed in 1\% formaldehyde at room temperature for $10 \mathrm{~min}$. After being washed, the cells were scraped and swelled in hypotonic swelling buffer, and then incubated on ice for $10 \mathrm{~min}$. Subsequently, the nuclei were lysed in SDS lysis buffer. IgG (Santa Cruz) and Antibodies against DNMT1 (Abcam) were used for IP. Real-time PCR was performed to amplify the DNMT1-binding region of the p53 promoters. The PCR primer sequences were: sense: 5'GGGTAAGTTTTTGATTGAATTTGAT-3' and antisense: 5'- CAAAACTCCACTCСТCTACCTAAAC-3'.

\section{Western blot}

Aggregate proteins in U87 and LN229 cells were extracted utilizing RIPA buffer (Radio-Immunoprecipitation assay buffer, Beyotime). Moreover, protein concentrations were quantified using BCA Protein Assay Kit Protein (Beyotime, Nantong, China). Equal quantities of samples were separated by utilizing 10 per cent SDS-PAGE gel before being added to the PVDF membrane (Millipore, Boston, MA, USA). Next, the membranes were blocked using the blocking solution for 1 hour and then incubated overnight at $4{ }^{\circ} \mathrm{C}$ using primary antibodies against DNMT1, p53 and GAPDH (Abcam, MA, USA). After being washed, corresponding HRP-conjugated secondary antibodies were used for incubation at room temperature for 2 hours. GAPDH was put to serve as an endogenous control. The images of protein bands were captured with the help of a Tanon detection system using ECL reagent (Thermo).

\section{Statistical analysis}

SPSS 22.0 (SPSS, Chicago, IL, USA) and Graphpad Prism 6.0 (GraphPad Software, La Jolla, CA, USA) were utilized to analyze the data. The differences between measured groups were analyzed via the Student $\mathrm{t}$ test. Data were presented as the mean \pm one standard deviation (SD). $\mathrm{P}<0.05$ was considered as statistically significant.

\section{Results}

\section{LINC00467 expression is remarkedly increased in glioma}

To investigate the expression of LINC00467 in human glioma, we searched the Cancer Genome Atlas (TCGA) database and found that the LINC00467 expression was significantly elevated in glioma tissues compared with normal tissue (Figure 1A). However, the results of TCGA database indicated that the expression level of LINC00467 was not associated with the overall survival rates of glioma patients (Figure 1B). The results suggested that LINC00467 may play a pivotal role in the progression of glioma.

\section{LINC00467 promoted glioma cell proliferation and invasion}

The expression levels of LINC00467 in glioma cell lines and negative control cells were detected using qRT-PCR. The results revealed that LINC00467 was upregulated in glioma cells compared with the controls, especially in U87 and LN229 cells (Figure 2A). We first constructed si-LINC00467 or pcDNALINC00467 and tested their transfection efficacies in glioma cells. We found that si-LINC00467 showed a better transfection efficacy in U87 cells (Figure 2B), and pcDNA-LINC00467 could markedly increase LINC00467 expression in LN229 cells (Figure 2C). 
After then, CCK8 and EdU assays were carried out to examine the effects of LINC00467 on cell proliferation capacity. As illustrated in Figure 2D-E, downregulation of LINC00467 significantly suppressed the growth of U87 cells, while upregulation of LINC00467 promoted cell proliferation of LN229 cells. To investigate the effects of LINC00467 on cell invasion,
Matrigel assays were performed with treated cell lines. And the results disclosed that knockdown of LINC00467 reduced the invasion activity of U87 cells, however, upregulation of LINC00467 significantly increased the number of invasive LN229 cells (Figure $2 \mathrm{~F})$.
A

D

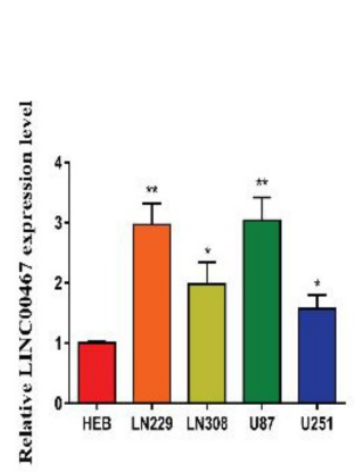

U87

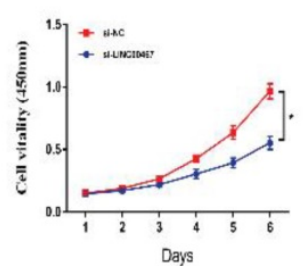

LN229

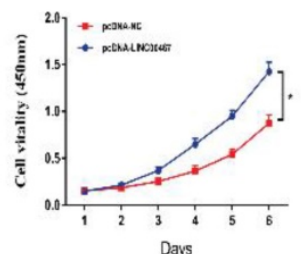

F
B

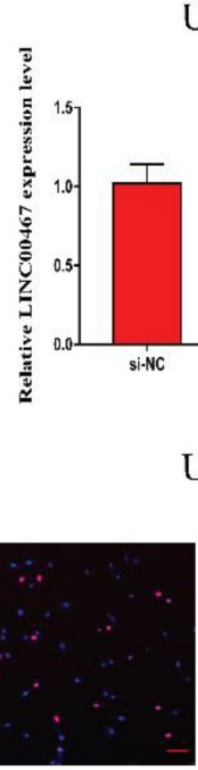

si-NC
U87

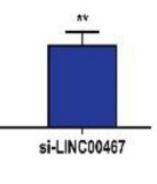

U87
C

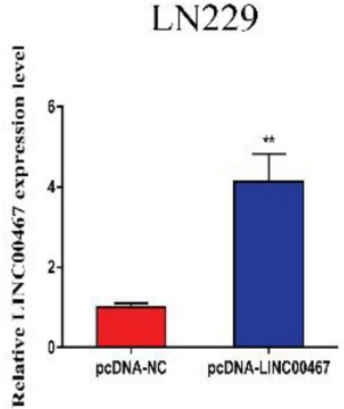

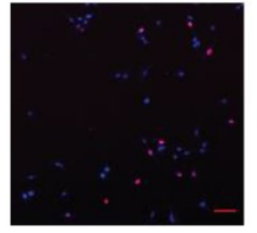

si-LINC00467

LN229

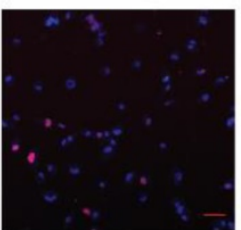

pcDNA-NC

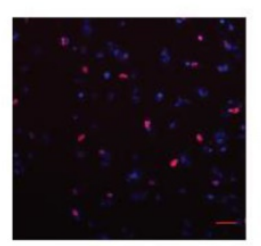

peDNA-LINC00467
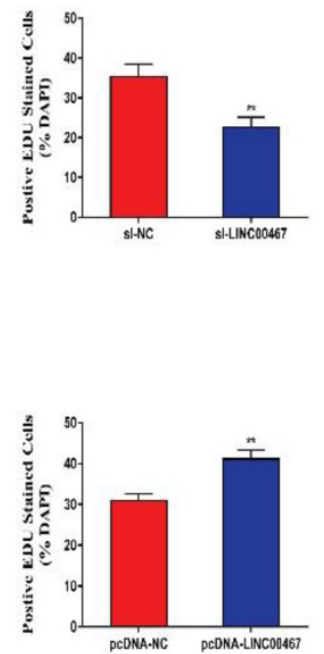

LN229

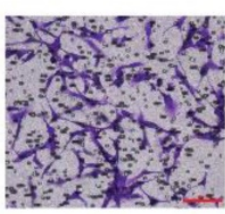

si-NC

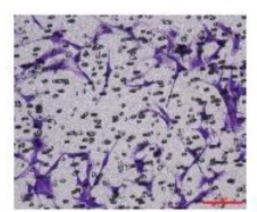

si-LINC00467 sin
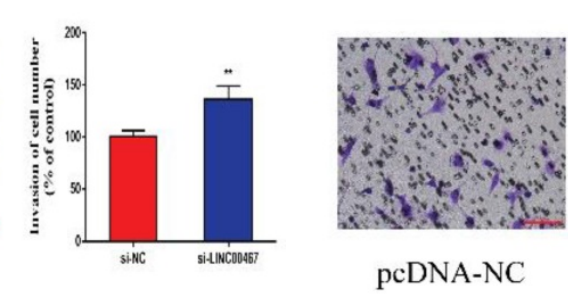

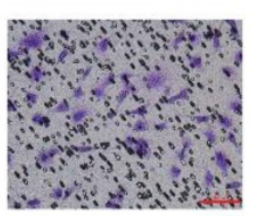

pcDNA-LINC00467

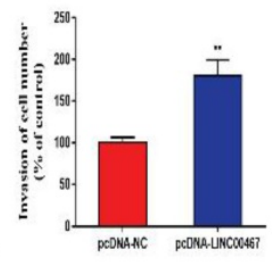

Figure 2. LINC00467 promoted glioma cell proliferation and invasion A. Quantitative real-time polymerase chain reaction (qRT-PCR) was used to analyse the expression of LINC00467 in human normal glial cell line (HEB) and glioma cells. B. QRT-PCR analysis of LINC00467 expression in si-NC, si-LINC00467 in U87 cells. C. QRT-PCR analysis of LINC00467 expression in pcDNA-NC, pcDNA-LINC00467 in LN229 cells. D. The CCK8 assay was used to determine the viability of si-LINC00467 transfected or pcDNA-LINC00467 transfected glioma cells. E. The EdU assay was used to determine the viability of si-LINC00467 transfected or pcDNA-LINC00467 transfected glioma cells. F. Downregulation of LINC00467 in U87 cells inhibited cell invasion while overexpression of LINC00467 in LN229 cells promoted cell invasive capability. The data represent the mean \pm SEM from three independent experiments. $* \mathrm{P}<0.05$, **P $<0.01$. CCK8, Cell-Counting Kit 8; EdU, Ethynyl deoxyuridine. 
A

U87

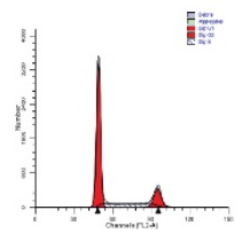

si-LINC00467

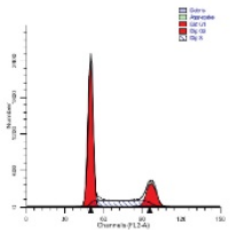

si-NC

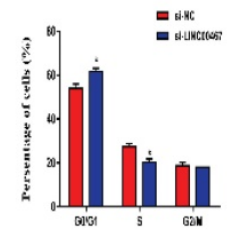

B

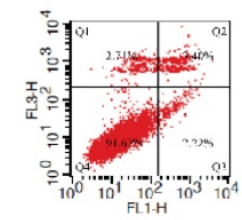

si-NC

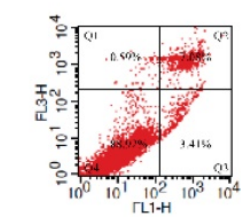

si-LINC00467

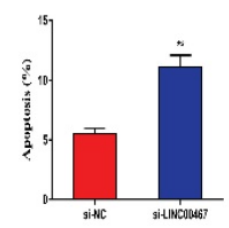

C
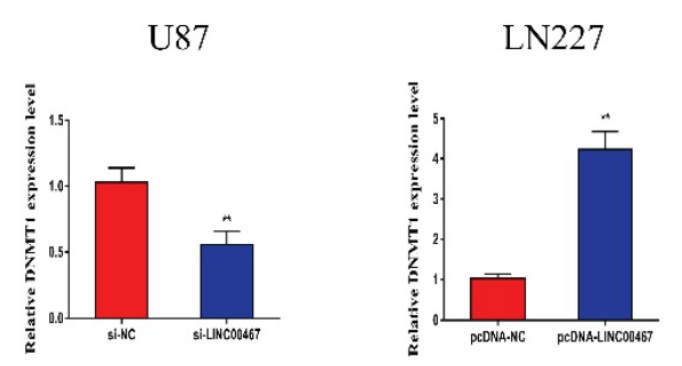

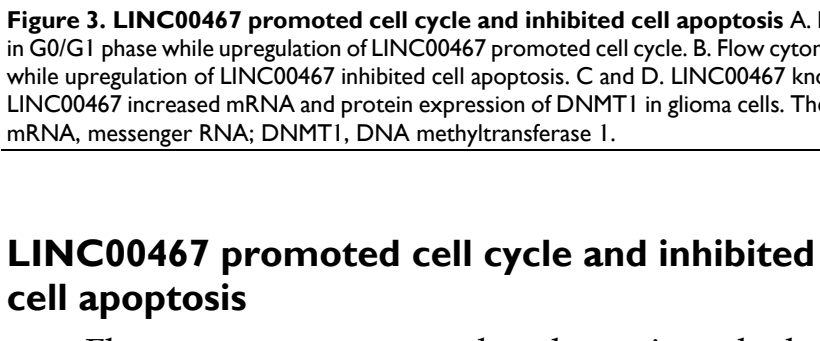

Flow cytometry was used to determine whether LINC00467 has an effect on cell cycle and apoptosis. As illustrated in Figure 3A, downregulation of LINC 00467 increased the G0/G1 arrest in U87 cells, while LN229 cells treated with pcDNA-LINC00467 reduced the proportion of G0/G1 phase. In addition, we also observed that knockdown of LINC00467 in U87 can induce cell apoptosis, while upregulation of LINC 00467 led to the opposite (Figure 3B). Previous study reported that LINC00467 might participate in cellular progress via positively regulating DNMT1 expression. Hence, we detected the expression of DNMT1 in glioma cells at both protein and mRNA levels. The results indicated that down-regulation of LINC00467 markedly reduced the expression of DNMT1; whilst up-regulation of LINC00467 increased the expression levels of DNMT1 (Figure 3C, 3D).

LN227

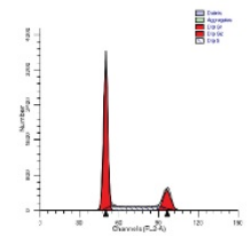

pcDNA-NC

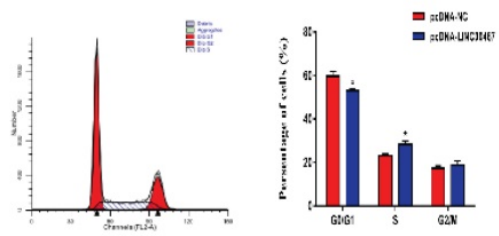

pcDNA-LINC00467

\section{LN227}

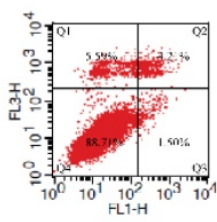

pcDNA-NC
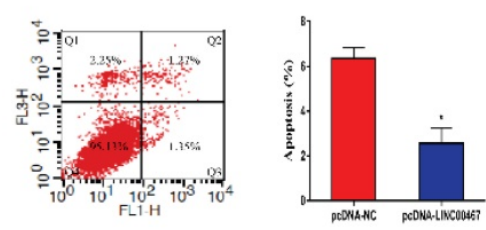

pcDNA-LINC00467

D
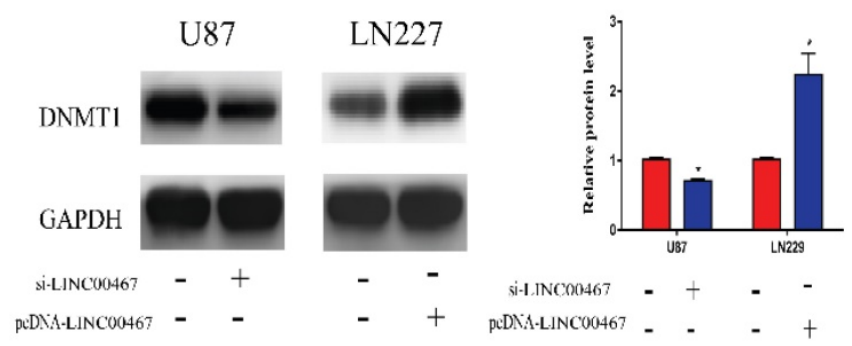

rested was used to detect apoptosis rates. Knockdown of LINC00467 promoted cell apoptosis data represent the mean \pm SEM from three independent experiments. ${ }^{* P}<0.05$, ${ }^{* *} \mathrm{P}<0.01$.
dassion of 
Many studies proved DNMT1 can bind to p53 promoter region and inhibit its expression. We hypothesized that LINC00467 may suppress p53 expression via binding to DNMT1. Then we investigated the p53 promoter region encompassing the 2000 bp upstream of the p53 transcription start site and identified 6 candidate $\mathrm{CpG}$ islands in this region (Figure S1). Then we conducted RIP and ChIP assays to verify the combination relationship. The results of RIP assay elucidated that LINC00467 could bind to DNMT1 in U87 and LN229 cells (Figure 4D). The ChIP assay demonstrated that DNMT1 could bind to DNAs in the p53 promoter region in glioma cells (Figure 4E).

A U87

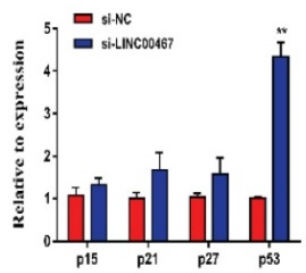

C
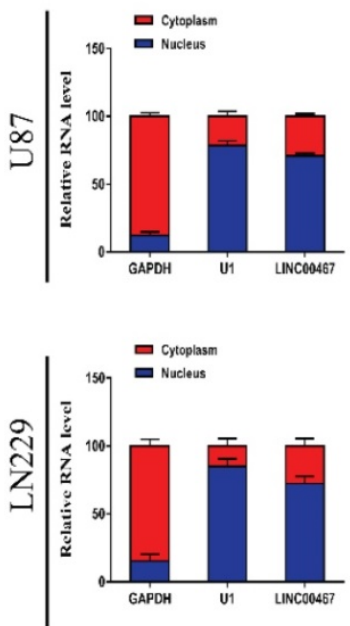

G U87

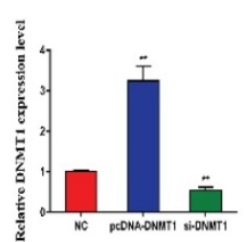

LN229

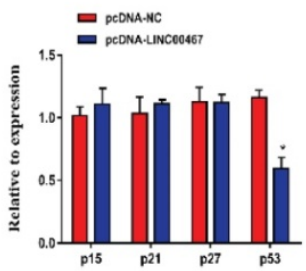

$\mathrm{D}$
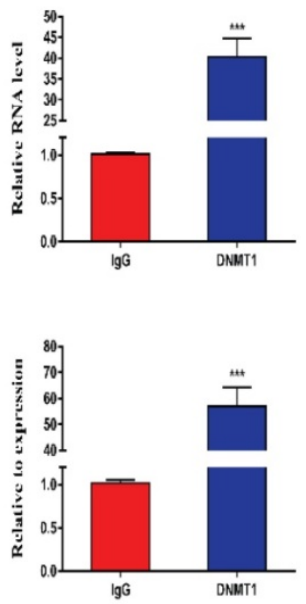

$\mathrm{H}$
LN229

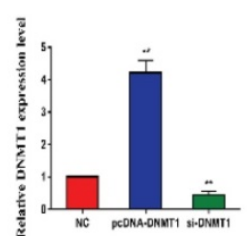

What's more, the binding levels of DNMT1 and p53 promoter was decreased when si-LINC00467 were transfected into the cells and upregulated with pcDNA-LINC00467 interference (Figure 4F). After cells transfected with si-DNMT1, the mRNA expression levels of DNMT1 were markedly downregulated, while DNMT1 expression was increased when cells transfected with pcDNA-DNMT1 (Figure 4G). The results of western blot assay showed that DNMT1 can negatively regulate p53 protein expression (Figure $4 \mathrm{H})$. All above results indicated that LINC00467 might inhibit p53 expression through binding to DNMT1.

B

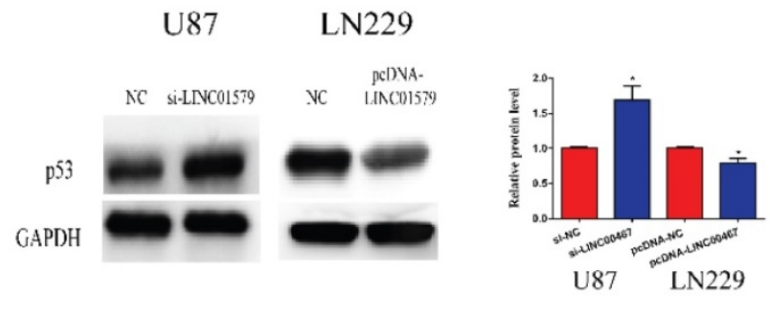

E

\section{F}
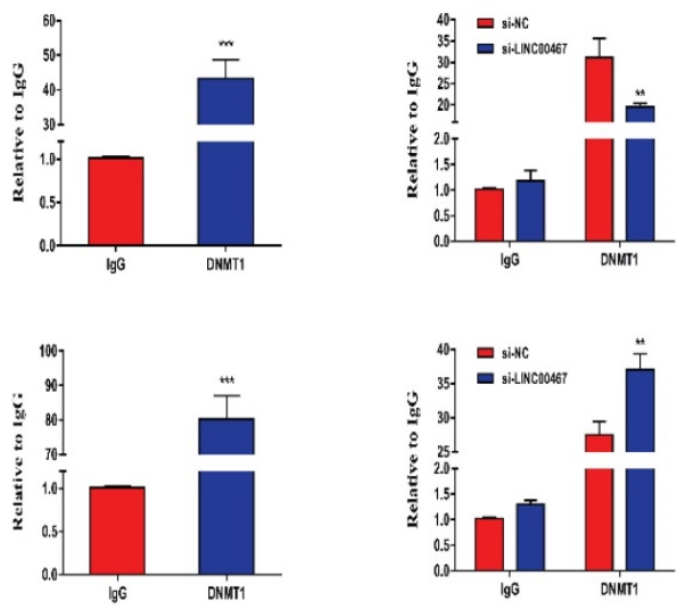

U87

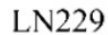

Figure 4. LINC00467 inhibited p53 expression by binding to DNMT1 A and B. LINC00467 knockdown upregulated mRNA and protein expression of p53 in U87 cells, while overexpression of LINC00467 suppressed mRNA and protein expression of p53 in LN229 cells. C. Nuclear-cytoplasmic separation assay showed that LINC00467 was mainly distributed in nuclear fractions of glioma cells. D. RIP results demonstrated that LINC00467 could be bound to DNMT1. E. ChIP results indicated that DNMT1 binds to DNAs in the $\mathrm{p} 53$ promoter region. F. Knockdown of LINC00467 in U87 cells downregulated the binding level of DNMT 1 and p53 promoter, while overexpression of LINC00467 in LN229 cells upregulated the binding level of DNMT1 and p53 promoter. G. Transfection efficacy of si-LINC00467 and pcDNA-LINC00467 in glioma cells. H. After interfering with si-DNMT1 in U87 cells, p53 expression significantly increased. Upregulation of DNMT1 in LN229 cells, p53 expression markedly decreased. The data represent the mean \pm SEM from three independent experiments. $* \mathrm{P}<0.05$, $* * \mathrm{P}<0.01$, $* * * \mathrm{P}<0.001$. mRNA, messenger RNA; DNMT1, DNA methyltransferase 1; RIP, RNA immunoprecipitation; CHIP, Chromatin immunoprecipitation. 
A
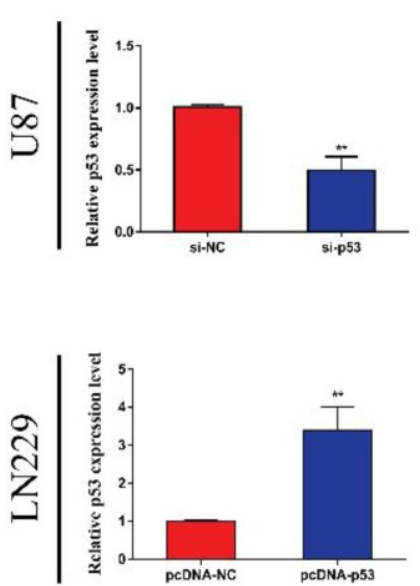

D

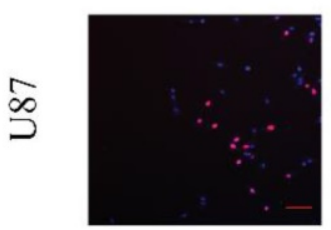

si-NC

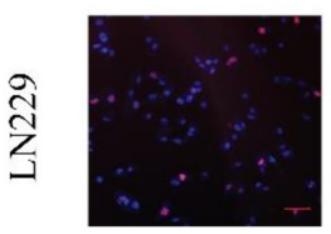

peDNA-NC

E

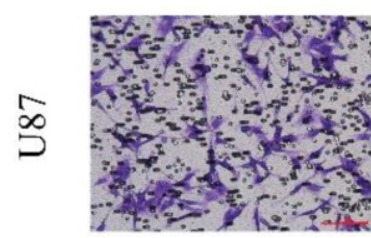

$\mathrm{si}-\mathrm{NC}$

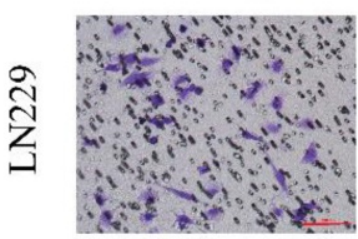

pcDNA-NC
B

$$
\text { p53 }
$$

$\mathrm{G} \Lambda \mathrm{PDH}$
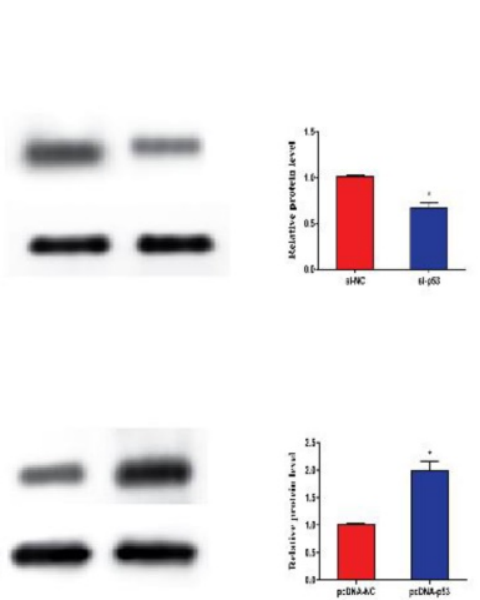

GAPDH
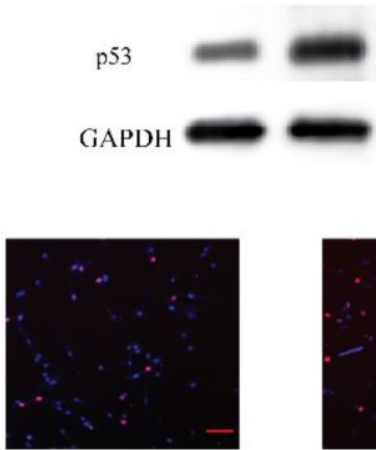

si-LINC01579

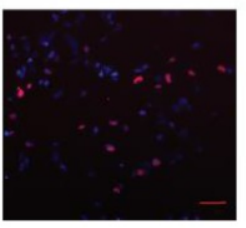

pcDNA-LINC01579

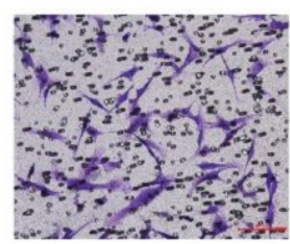

si-LINC01579

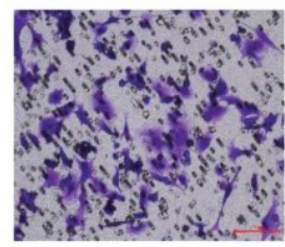

peDNA-LINC01579

si-LINC01579

- si-p53

pcDNA-LINC01579 + pcDNA-p53

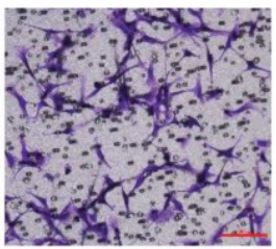

si-LINC01579

si-p53

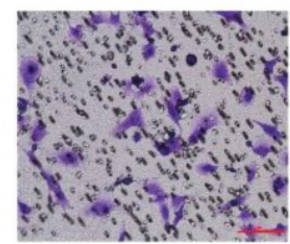

pCDNA-LINC01579
C
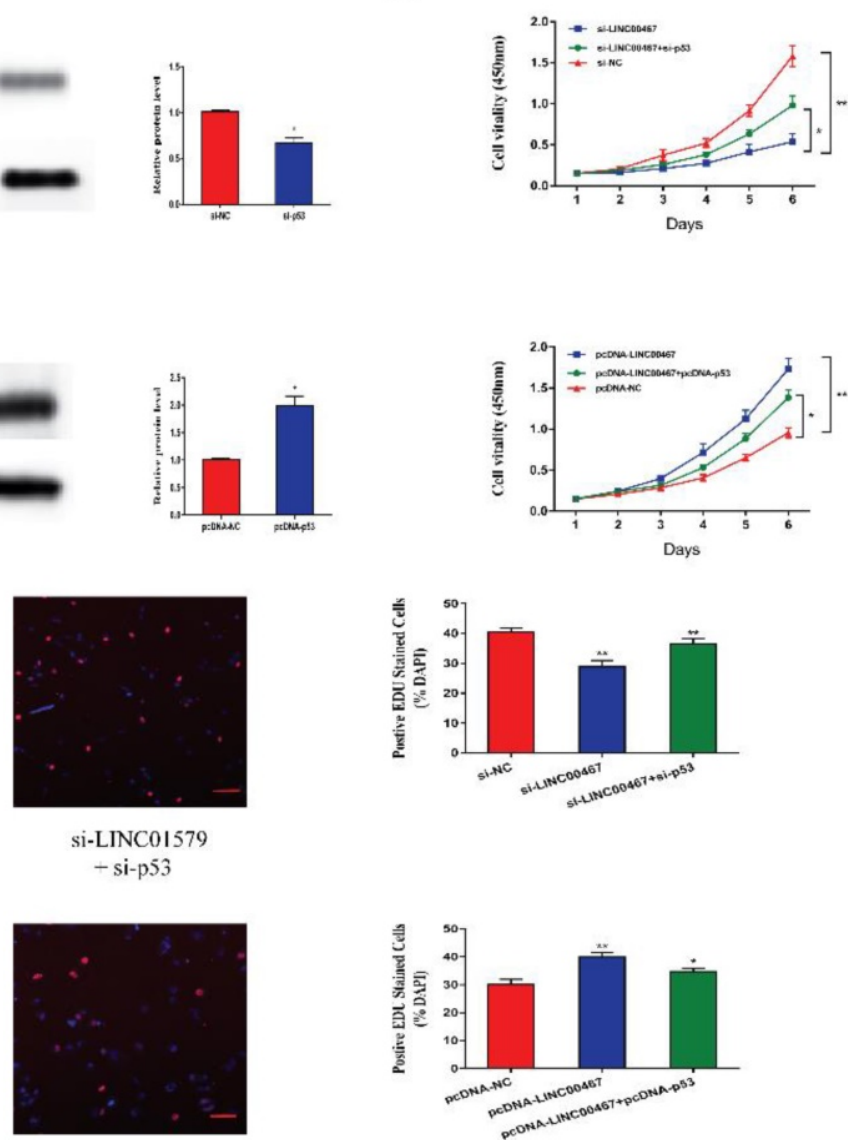

+ peINA-p53

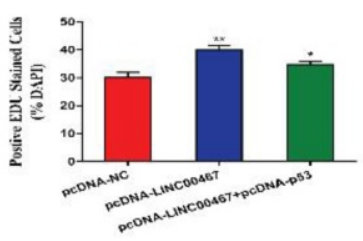

Figure 5. P53 reversed the anti-tumor effect of LINC00467 on glioma A, B. After transfection of si-p53 and pcDNA-p53 in glioma cells, mRNA and protein levels of $p 53$ correspondingly changed. C and D. Cell proliferative ability was significantly decreased after downregulation of LINC00467, which was reversed by knockdown of p53. Similarly, cell proliferative ability could be enhanced after overexpression of LINC00467 and reversed by overexpression of p53. E. Cell invasive ability was significantly suppressed after knockdown of LINC00467, which was reversed by downregulation of p53. Moreover, cell invasive ability was significantly enhanced after overexpression of LINC00467, which was reversed by overexpression of $\mathrm{p} 53$. The data represent the mean \pm SEM from three independent experiments. *P $<0.05$, **P $<0.01$. mRNA, messenger RNA.

\section{P53 reversed the effects of LINC00467 on cell proliferation and metastasis}

We transfected glioma cells with si-p53 or pcDNA-p53 and performed qRT-PCR and Western blot assay to test the transfection efficiency. The results showed that si-p53 could reduce the mRNA and protein levels of p53, while pcDNA-p53 could upregulate the expression of p53 (Figure $5 \mathrm{~A}$ and $5 \mathrm{~B}$ ). Then we performed CCK8 and EdU assays to detect 
cell proliferative ability. We found that cell proliferation was markedly attenuated after downregulation of LINC00467, which was partially reversed after cotransfected with si-p53 in U87 cells. Moreover, the ability of cell proliferation was significantly enhanced after overexpression of LINC00467, which was reversed by overexpression of p53 (Figure 5C, D). Similarly, the effect of LINC00467 on cell invasion ability could be reversed by p53 (Figure 5E). These findings indicated that LINC00467 could foster cell growth and invasion by inhibiting the expression of p53.

\section{Discussion}

Malignant gliomas, with high risk of invasion and high mortality, are considered as the most common form of primary intrinsic brain tumors around the world $[18,19]$. LncRNAs are regarded as critical roles in the pathogenesis of glioma [20]. Many IncRNAs have been identified to be dysregulated in glioma patients, which may contribute to glioma abnormal growth and metastasis. For instances, lncRNA metastasis-associated lung adenocarcinoma transcript 1 (lncMALAT1) acts as a tumor-suppressive role in glioma by downregulating matrix metallopeptidase 2 (MMP2) and inactivating the ERK/MAPK signaling [21], upregulation of lncHOXA-AS3 in glioma fosters tumor progression and indicates poor prognosis [22], lncRNA colon cancer-associated transcript 2 (lncCC AT2) is highly expressed in glioma and can promote cell growth and migration [23]. Given that current standard treatments, including surgery, radiotherapy and traditional chemotherapy, barely have any impact on the general prognosis of patients with glioma [24, 25], alternative therapeutic strategies are needed to be explored.

Previous studies have demonstrated that LINC 00467 was upregulated in various types of malignant tumors, which contributed to tumorigenesis through different kinds of pathways. However, the functional roles and potential mechanism of LINC00467 in glioma tumorigenesis remain unknown. In this study, LINC00467 was found to be abnormally expressed in glioma through the detection database. Then we also discovered that LINC00467 is markedly upregulated in glioma cell lines compared with negative control cells (HEB). What's more, the results of cell function assays revealed that the upregulation of LINC00467 significantly promoted cell growth, invasion and accelerated cell cycle and inhibited cell apoptosis in glioma cells.

As is known to us, lncRNAs could regulate cellular biological progress at different levels including transcription, post-transcriptional modifycations and translation [26, 27]. Hence, we performed experiments and found that LINC00467 mainly located in nucleus of glioma cells, which meant that LINC00467 might play its function at the transcriptional level. Wang et al., reported that LINC00467 contributes to lung adenocarcinoma cell proliferation and metastasis via binding with EZH2 and repressing HTRA3 expression [17]. Accumulating evidence has demonstrated that lncRNAs could bind to transcription factors to take a part in target gene regulation [28]. Then RIP assay was performed to verify the combination relationship between LINC00467 and DNMT1. The results of experiments elucidated that DNMT1 could bind to LINC00467, and LINC00467 was capable of stabilizing DNMT1 expression. DNMT1 is the primary enzyme that maintains the methylation pattern onto daughter strands after DNA replication [29]. In many tumor cells, including glioma cells, high methylation of tumor-suppressor genes and abnormal cell proliferation and apoptosis are associated with increased DNMT1 activity [30]. Previous studies proved that DNMT1 can bind to p53 promoter region and inhibit its expression in glioma $[31,32]$. Tumor suppression is as a cellular defense mechanism, tumor suppression can prevent normal cells from neoplastic transformation, and p53, a tumor suppressor, serves as an essential component in the initiation and progression of glioma [33]. Hence, CHIP assay and western blot assay were performed to verify the binding condition between LINC00467, DNMT1 and p53. As expected, DNMT1 was able to bind to p53 and inhibited p53 expression, while LINC 00467 could suppress p53 expression via binding to DNMT1. Then, CCK8 and EdU assays, transwell invasion assays were performed to verify the effect of LINC00467 on p53. All the results of experiments demonstrated that p53 could reverse the impact of LINC00467 on glioma cells, which meant that LINC 00467 may promote cell proliferation and invasion by reducing p53 expression.

\section{Conclusions}

In conclusion, this study revealed that high expression of LINC00467 can promote cell growth and invasion and inhibit the apoptosis of glioma cells, potentially via the inhibition of p53 expression by binding to DNMT1.

\section{Supplementary Material}

Supplementary figure.

http://www.jcancer.org/v11p2935s1.pdf

\section{Acknowledgements}

This work was supported by the Science and Technology Development Fund of Nanjing Medical University [Grant No. NMUB2018281]. 


\section{Author Contributions}

Yin Zhang, Xuefeng Jiang, Huamin Tang and Hongyi Liu designed this study; Yin Zhang, Daling $\mathrm{Hu}$, Junli Jia and Jialin Yao conducted the experiments; Jinfeng Guo and Tian Tang served as scientific advisors, Xuefeng Jiang and Zhisheng $\mathrm{Wu}$ participated in writing or technical editing of the manuscript.

\section{Competing Interests}

The authors have declared that no competing interest exists.

\section{References}

1. Bush NA, Chang SM, Berger MS. Current and future strategies for treatment of glioma. Neurosurg Rev. 2017; 40: 1-14.

2. Ohgaki H, Kleihues $P$. Genetic pathways to primary and secondary glioblastoma. Am J Pathol. 2007; 170: 1445-53.

3. Guillamo JS, Monjour A, Taillandier L, Devaux B, Varlet P, Haie-Meder C, et al. Brainstem gliomas in adults: prognostic factors and classification. Brain. 2001; 124: 2528-39.

4. Ryall S, Tabori U, Hawkins C. A comprehensive review of paediatric low-grade diffuse glioma: pathology, molecular genetics and treatment. Brain Tumor Pathol. 2017; 34: 51-61.

5. Liu K, Jiang Y. Polymorphisms in DNA Repair Gene and Susceptibility to Glioma: A Systematic Review and Meta-Analysis Based on 33 Studies with 15 SNPs in 9 Genes. Cell Mol Neurobiol. 2017; 37: 263-74.

6. Ba Z, Gu L, Hao S, Wang X, Cheng Z, Nie G. Downregulation of IncRNA CASC2 facilitates osteosarcoma growth and invasion through miR-181a. Cell Prolif. 2018; 51.

7. Wang G, Pan J, Zhang L, Wei Y, Wang C. Long non-coding RNA CRNDE sponges miR-384 to promote proliferation and metastasis of pancreatic cancer cells through upregulating IRS1. Cell Prolif. 2017; 50.

8. Fu M, Huang Z, Zang X, Pan L, Liang W, Chen J, et al. Long noncoding RNA LINC00978 promotes cancer growth and acts as a diagnostic biomarker in gastric cancer. Cell Prolif. 2018; 51.

9. Peng L, Chen G, Zhu Z, Shen Z, Du C, Zang R, et al. Circular RNA ZNF609 functions as a competitive endogenous RNA to regulate AKT3 expression by sponging miR-150-5p in Hirschsprung's disease. Oncotarget. 2017; 8: 808-18.

10. Cao C, Zhang T, Zhang D, Xie L, Zou X, Lei L, et al. The long non-coding RNA, SNHG6-003, functions as a competing endogenous RNA to promote the progression of hepatocellular carcinoma. Oncogene. 2017; 36: 1112-22.

11. Chen $Z$, Chen $X$, Chen P, Yu S, Nie F, Lu B, et al. Long non-coding RNA SNHG20 promotes non-small cell lung cancer cell proliferation and migration by epigenetically silencing of P21 expression. Cell Death Dis. 2017; 8: e3092.

12. Mazar J, Rosado A, Shelley J, Marchica J, Westmoreland TJ. The long non-coding RNA GAS5 differentially regulates cell cycle arrest and apoptosis through activation of BRCA1 and p53 in human neuroblastoma. Oncotarget. 2017; 8: 6589-607.

13. Han D, Gao X, Wang M, Qiao Y, Xu Y, Yang J, et al. Long noncoding RNA H19 indicates a poor prognosis of colorectal cancer and promotes tumor growth by recruiting and binding to eIF4A3. Oncotarget. 2016; 7: 22159-73.

14. Cai K, Li T, Guo L, Guo H, Zhu W, Yan L, et al. Long non-coding RNA LINC00467 regulates hepatocellular carcinoma progression by modulating miR-9-5p/PPARA expression. Open Biol. 2019; 9: 190074.

15. Yang J, Liu Y, Mai X, Lu S, Jin L, Tai X. STAT1-induced upregulation of LINC00467 promotes the proliferation migration of lung adenocarcinoma cells by epigenetically silencing DKK1 to activate Wnt/beta-catenin signaling pathway. Biochem Biophys Res Commun. 2019; 514: 118-26.

16. Atmadibrata B, Liu PY, Sokolowski N, Zhang L, Wong M, Tee AE, et al. The novel long noncoding RNA linc00467 promotes cell survival but is down-regulated by N-Myc. PLoS One. 2014; 9: e88112.

17. Wang $X$, Liu $\mathrm{H}$, Shen $\mathrm{K}$, Pan $\mathrm{X}$, Wei $\mathrm{Y}, \mathrm{Lv} \mathrm{T}$, et al. Long intergenic noncoding RNA 00467 promotes lung adenocarcinoma proliferation, migration and invasion by binding with EZH2 and repressing HTRA3 expression. Mol Med Rep. 2019; 20: 640-54

18. Chen J, Li Y, Yu TS, McKay RM, Burns DK, Kernie SG, et al. A restricted cell population propagates glioblastoma growth after chemotherapy. Nature. 2012; 488: 522-6.

19. Del Vecchio CA, Li G, Wong AJ. Targeting EGF receptor variant III: tumor-specific peptide vaccination for malignant gliomas. Expert Rev Vaccines. 2012; 11: 133-44.

20. Reon BJ, Anaya J, Zhang Y, Mandell J, Purow B, Abounader R, et al. Expression of lncRNAs in Low-Grade Gliomas and Glioblastoma Multiforme: An In Silico Analysis. PLoS Med. 2016; 13: e1002192.

21. Han $\mathrm{Y}, \mathrm{Wu} \mathrm{Z}, \mathrm{Wu} \mathrm{T}$, Huang $\mathrm{Y}$, Cheng $\mathrm{Z}, \mathrm{Li} \mathrm{X}$, et al. Tumor-suppressive function of long noncoding RNA MALAT1 in glioma cells by downregulation of MMP2 and inactivation of ERK/MAPK signaling. Cell Death Dis. 2016; 7 : e2123

22. Wu F, Zhang C, Cai J, Yang F, Liang T, Yan X, et al. Upregulation of long noncoding RNA HOXA-AS3 promotes tumor progression and predicts poor prognosis in glioma. Oncotarget. 2017; 8: 53110-23.

23. Guo H, Hu G, Yang Q, Zhang P, Kuang W, Zhu X, et al. Knockdown of long non-coding RNA CCAT2 suppressed proliferation and migration of glioma cells. Oncotarget. 2016; 7: 81806-14.

24. Van Meir EG, Hadjipanayis CG, Norden AD, Shu HK, Wen PY, Olson JJ. Exciting new advances in neuro-oncology: the avenue to a cure for malignant glioma. CA Cancer J Clin. 2010; 60: 166-93.

25. Zhang L, Wu X, Xu T, Luo C, Qian J, Lu Y. Chemotherapy plus radiotherapy versus radiotherapy alone in patients with anaplastic glioma: a systematic review and meta-analysis. J Cancer Res Clin Oncol. 2013; 139: 719-26.

26. Tsai MC, Manor O, Wan Y, Mosammaparast N, Wang JK, Lan F, et al. Long noncoding RNA as modular scaffold of histone modification complexes. Science. 2010; 329: 689-93.

27. Wilusz JE, Freier SM, Spector DL. $3^{\prime}$ end processing of a long nuclear-retained noncoding RNA yields a tRNA-like cytoplasmic RNA. Cell. 2008; 135: 919-32.

28. Gonzalez I, Munita R, Agirre E, Dittmer TA, Gysling K, Misteli T, et al. A IncRNA regulates alternative splicing via establishment of a splicing-specific chromatin signature. Nat Struct Mol Biol. 2015; 22: 370-6.

29. Zhang P, Sun H, Yang B, Luo W, Liu Z, Wang J, et al. miR-152 regulated glioma cell proliferation and apoptosis via Runx2 mediated by DNMT1. Biomed Pharmacother. 2017; 92: 690-5.

30. Guo Z, Li G, Bian E, Ma CC, Wan J, Zhao B. TGF-beta-mediated repression of MST1 by DNMT1 promotes glioma malignancy. Biomed Pharmacother. 2017; 94: 774-80.

31. Georgia S, Kanji M, Bhushan A. DNMT1 represses p53 to maintain progenitor cell survival during pancreatic organogenesis. Genes Dev. 2013; 27: 372-7.

32. Yang J, Platt LT, Maity B, Ahlers KE, Luo Z, Lin Z, et al. RGS6 is an essential tumor suppressor that prevents bladder carcinogenesis by promoting p53 activation and DNMT1 downregulation. Oncotarget. 2016; 7: 69159-72.

33. Li J, Bian EB, He XJ, Ma CC, Zong G, Wang HL, et al. Epigenetic repression of long non-coding RNA MEG3 mediated by DNMT1 represses the p53 pathway in gliomas. Int J Oncol. 2016; 48: 723-33. 